\title{
An Investigation of Antibiotic Prescribing in Patients with Upper Respiratory Tract Infections (Urtis) at Katutura Health Centre, Windhoek, Namibia
}

\section{Mwape Kunda ${ }^{1}$, Lischen Haoses-Gorases ${ }^{2 *}$ and Marcus Goraseb ${ }^{3}$}

${ }^{1}$ Masters of Public Health, Ministry of Health and Social Services, Namibia

${ }^{2} \mathrm{PhD}$ in Nursing Science, Faculty of Health Sciences, University of Namibia, Namibia

${ }^{3} \mathrm{MPH}, \mathrm{MD}$ School of Medicine, University of Namibia, Namibia

\begin{abstract}
Upper respiratory tract infections (URTIs) are of viral cause in $80 \%$ of cases and they constitute a major part of the primary health care practitioners' workload with antibiotics commonly prescribed for these illnesses. The aim of this study was to explore antibiotic prescribing in patients with URTIs at Katutura Health Centre, Namibia. A descriptive, cross sectional, quantitative design based on patients' prescriptions was used. Data were analyzed using Epi info statistical package version 7.1.1.14. The rate of antibiotic prescribing for patients with URTIs at Katutura Health Centre (KHC) was 78\% (95\% Cl, 74\%-82\%). Further bivariate analyses of antibiotic prescribing (Response variable) and illness, age, gender and profession (exposure variables) showed that age and illness were significantly associated with antibiotic prescribing $(p<0.05)$ while gender and profession rank were not. In conclusion, the Ministry of Health and Social Services need to develop or adopt international strategies have proven efficacy in reducing antibiotic prescribing in URTIs
\end{abstract}

Keywords: Investigation; Antibiotics; Patients; Upper respiratory tract infections; Infections; Rationality; Compliance

\section{Introduction}

Patients with Upper Respiratory Tract Infections (URTIs) constitute a major part of the primary health care practitioners' workload, especially during winter [1] and as indicated by Kontarakis et al. [2], primary health care practitioners' account for the majority of antibiotic prescribing. Mazur [3] indicated that these infections are viral in $80 \%$ of the cases and do not respond to antibiotic therapy. In spite of this, physicians continue to prescribe antibiotics even though they know that antibiotics are not effective against viral infections [4]. A study done in Turkey indicated that antibiotics were prescribed for $91.8 \%$ of patients with URTIs. The same study also showed that antibiotics were prescribed for $41.9 \%$ of patients with a common cold, $94.7 \%$ of patients with acute pharyngitis, $94.1 \%$ of patients with acute sinusitis and $100 \%$ of those with acute otitis media [5]. The World Health Organization [6] also estimated that up to $60 \%$ of people with viral upper respiratory tract infection receive antibiotics inappropriately.

Considering these figures, it is clear that antibiotic prescribing in URTIs (among others) is alarmingly high and contributes to the emergence and spread of bacterial resistance, which is a major global public health problem. This worldwide epidemic of antibiotic resistance is in danger of ending the golden age of antibiotic therapy [7]. Antibiotic resistance leads to a decrease in treatment success which results in increased mortality, morbidity, and cost of health care [8]. For instance, the rapid increase in drug-resistant Streptococcus pneumonia infections are of particular concern in paediatric patients since pneumococci is the leading cause of bacterial meningitis, pneumonia, bacteraemia and otitis media in children [9]. Furthermore, infections caused by antimicrobial-resistant bacteria are associated with substantially higher rates of morbidity and mortality compared to infections caused by antimicrobial-susceptible bacteria [10].

Irrational use of antibiotics also leads to wastage of resources, adverse effects which need more resources to be treated and patient misconception of these medicines, among others. It is against this background that the current study aimed to explore antibiotic prescribing in patients with URTIs by determining the rate of antibiotic prescribing in patients with URTIs, assessing the rational treatment of URTIs and comparing the costs of rational and irrational antibiotic use of URTIs.

The purpose of this study was to explore antibiotic prescribing in patients with URTIs at Katutura Health Centre (KHC). The objective were to determine the rate of antibiotic prescribing in patients with URTIs, to assess the rationality of the treatment of URTIs and to compare the cost of rational and irrational antibiotic use in URTIs.

\section{Literature Review}

URTIs are viral in $80 \%$ of cases [3] but antibiotic prescribing in these illnesses still remains a problem in many places where about $60 \%$ of patients with URTIs receive antibiotics inappropriately [6]. There has been no study done in Namibia to determine the rate of antibiotic prescribing in URTIs, but results from PMIS by Phulu, Sumbi and Lates [11], show that many facilities in Namibia, Katutura Health Centre (KHC) inclusive do not achieve the target of the indicator HF13 that monitors antibiotic prescribing in outpatients. This problem could be attributed to antibiotic prescribing in URTIs because they constitute a major part of the primary health care practitioners' workload [1]. Irrational antibiotic prescribing is a global public health problem and according to Harris et al. [12] URTIs are important targets for strategies aimed at reducing excess antibiotic use because antibiotics are frequently prescribed in these illnesses that are predominantly of viral etiology.

*Corresponding author: Lischen Haoses-Gorases, PhD in Nursing Science Faculty of Health Sciences, University of Namibia, Tel: +264 612063111 E-mail: Ihaoses@unam.na (or) 2002@iway.na

Received August 22, 2015; Accepted October 17, 2015; Published October 19 2015

Citation: Kunda M, Haoses-Gorases L, Goraseb M (2015) An Investigation of Antibiotic Prescribing in Patients with Upper Respiratory Tract Infections (Urtis) at Katutura Health Centre, Windhoek, Namibia. Single Cell Biol 4: 122 doi:10.4172/2168-9431.1000122

Copyright: (c) 2015 Salvatore F, et al. This is an open-access article distributed under the terms of the Creative Commons Attribution License, which permits unrestricted use, distribution, and reproduction in any medium, provided the original author and source are credited. 
Though URTIs are viral in most cases, rational prescribing is important in cases requiring antibiotic treatment in order to avoid development of resistance and treatment failure. According to WHO [13] rational prescribing refers to patients receiving medications appropriate to their clinical needs (right drug), in doses that meet their own individual requirements, for an adequate period of time, and at the lowest cost to them and their community. Therefore the assessment of rationality of antibiotic prescriptions in this study was based on Namibian Standard Treatment Guidelines (NSTGs) and WHO recommendations. These STGs recommend antibiotic prescribing in all the conditions that were included in the study except for the common cold. STG's are systematically developed statements to help practitioners or prescribers to make decisions about appropriate treatments for specific clinical conditions. They include information such as; diagnostic criteria, treatment of first choice (drug choice, dose, duration), important side effects, contraindications, referral criteria, and also medicine information for patients [14]. In this study, the aspects that were assessed were the choice of antibiotic, dose, frequency and duration of treatment. The upper respiratory infections referred to in this study are the common cold, pharyngitis, tonsillitis, acute otitis media, and tonsillitis, [15] which significantly occur in the upper respiratory tract. URTI are regarded as nonspecific, a term that is used to describe acute infections involving the nose, paranasal sinuses, pharynx, larynx, trachea and bronchi. Hence, proper judgment is required in determining the affected respiratory mucosal part. URTIs are caused by viruses in most cases, but they can also be bacterial in etiology. The symptoms are usually mild and self-limiting, but could lead to life threatening conditions if they are caused by bacteria or if a bacterial infection follows a viral infection. The symptoms that include stuffy nose, fever, vomiting, and irritability, loss of appetite, watery eyes, runny nose, sneezing, coughing and sore throat last for 7 to 10 days or longer [16]. URTIs affect both children and adults and are a major cause of mild morbidity which has a high cost to society, being responsible for missed work and unnecessary medical care. Occasionally they have serious sequelae [17].

\section{Methods}

A quantitative, cross-sectional descriptive design was chosen as the most appropriate for the study in order to maximize the validity of the research results [18]. The population comprised of all prescriptions of patients diagnosed with URTIs, specifically diagnosed with common cold, pharyngitis, tonsillitis and pharyngitis and tonsillitis at KHC during 2013. In this study the sample comprised of those patients diagnosed with URTI's that met inclusion criteria for patients with only a single diagnosis from the following list: common cold, pharyngitis, tonsillitis, pharyngitis, pharyngitis and Tonsillitis (pharyngeotonsillities), AOM, and URTI. It also included newly diagnosed acute infections. All diagnoses were considered acute unless specified as chronic. The appropriate sample size was calculated as follows:-

$$
\begin{aligned}
& \mathrm{n}=\mathrm{z}_{(1-\alpha / 2)}^{2} \times \mathrm{p}(\mathrm{p}-1) \div \mathrm{d}^{2} \\
& \text { Sample size }(\mathrm{n})=\frac{1.96^{2} \times(0.5) \times(1-0.5)}{0.05^{2}}=385 .
\end{aligned}
$$

Therefore the calculated sample size was 385 for KHC.

\section{Sample size}

Since it's usually not feasible to study a whole population, a sample of the population is drawn that is statistically representative of the population so that from the results based on the sample inference to the population can be made.

By definition, a sample is a part or a fraction of a whole, or a subset of a larger set selected by the researcher to participate in a research study. It consists of a selected group of elements or units of analysis from a defined population [18]. The sample comprised of prescriptions of URTIs that met the eligibility criteria. The appropriate sample size was calculated as follows:

$$
\mathrm{n}=z_{(1-\alpha / 2)}^{2} \times p(p-1) \div d^{2}[19] .
$$

Where:

The value of $Z_{(1-\alpha / 2) \text { is }}$ taken from the standard normal distribution table, $p$ is the expected prevalence or proportion and $d$ is the precision.

- For a confidence interval of $95 \%$, the $\mathrm{Z}$-value is 1.96 ;

- Expected proportion ( $\mathrm{p})$. This is the proportion the investigator aims to estimate by doing the study. In many cases, this can be derived either from previous studies or by doing a pilot study. It is also suggested that if there was doubt about the value of ' $\mathrm{p}$ ', it is best to err towards $50 \%$ as it would lead to a larger sample size; and

- Precision (d), the level of precision, sometimes called sampling error, is the range in which the true value of the population is estimated to be. This range is often expressed in percentage points, (e.g., +-5\%). At the end of the study, the prevalence is presented with its $95 \%$ confidence interval. E.g., if the prevalence is $20 \%$ and $95 \%$ CL is $10 \%$ to $30 \%$. It means that the study has estimated the population prevalence is between $10 \%$ and $30 \%$. The precision (d) for this estimate is $10 \%$ (i.e. $20 \%+-$ $10 \%=10 \%-30 \%)$. It shows that the width of the confidence interval is two times the precision $(\mathrm{cl}=2 \mathrm{~d})$.

The convenience sampling method was used to select 385 prescriptions of all patients that were seen at KHC during this period and met the eligibility criteria. The instrument was also piloted prior to the actual data collection that revealed that practitioners sometimes record two diagnoses, e.g. pharyngitis and tonsillitis that made it difficult to judge the basis upon which antibiotics were prescribed. In addition, patients seen with recurrent or non-resolving URTIs were more likely to have antibiotics prescribed.

The following inclusion criteria were applied:

Patients with only a single diagnosis from the following list that is common cold, pharyngitis, tonsillitis, pharyngitis, pharyngitis and tonsillitis (pharyngeotonsillities), Acute Otitis Media (AOM) and URTI, newly diagnosed acute infections.

All diagnoses were considered acute unless specified as chronic. Only prescriptions of patients diagnosed with a URTI were included in the study. The following exclusion criteria were applied:

Prescriptions with a chronic or recurrent diagnosis of the conditions under study, prescriptions having a diagnosis other than those under study and prescriptions from other facilities apart from Katutura Health Centre.

The actual data collection only commenced after obtaining approval from the University of Namibia (UNAM) research review committee and MoHSS. In addition, the research assistant was trained before starting the collection of data.

Assessment of medicines use can be made by extraction of the 
required information from patient's prescriptions [20]. In this study, patients' prescriptions (health passports) were used to obtain the required data which was entered on a data collection form. This form was developed to contain all variables of interest. The variables being, patient age and gender, diagnosis, antibiotic prescribed, quantities of antibiotics dispensed, compliance to STGs of antibiotic prescriptions, other medicines prescribed and prescriber rank or profession In order to ensure confidentiality and anonymity of both the prescribers and the patients, their names were not recorded on the extraction form. Instead, all data extraction forms were allocated an identification number from 1 to 385 and only the prescriber's profession was recorded. Data were collected on patient age, gender, diagnosis, treatment of the condition (whether antibiotics were prescribed or not), names of antibiotics prescribed, compliance with guidelines, the number of antibiotics prescribed, other medicines prescribed, total number of medicines prescribed, quantity dispensed of antibiotics and profession rank or position of the prescriber. In addition, data were collected on the total number of medicines prescribed per prescription. Data collection was undertaken from KHC main pharmacy by the researcher and one assistant on every working day of the week from the $18^{\text {th }}$ of May 2013 to the $24^{\text {th }}$ of July 2013. At the end of each data collection day, the researcher checked all the filled forms for completeness and also assessed treatment appropriateness, after which the section on treatment appropriateness/ rationality was filled. All the filled forms were kept by the researcher and all the data elements on the extraction form were entered on Epi-Info version 7: 1.1.14 for analysis. Content and face validity were considered in this study. This was ensured by having the tool reviewed by the research supervisors and statistician and by conducting a pilot study. After which the content of the instrument was refined to contain only variables of interest. Face validity refers to the extent to which the tool appears to be addressing the variables or concepts of interest (Sim and Wright). This was ensured by peer review and review by research supervisors and statistician.

\section{Results}

\section{Antibiotic prescribing in URTIs}

Data from 385 prescriptions were collected and analyzed by using Epi Info version 7.1.1.14 and statistical Package for Social Sciences (SPSS) version 22.0 statistical package Microsoft Office was also used to present graphical data (Table 1).

The sample comprised of 55\% $(n=213)$ prescriptions for female patients and $45 \%(\mathrm{n}=172)$ for male patients. In addition, prescriptions of patients aged 5 and below made up the largest proportion of the sample, that is $34 \%(n=131)$ (Figure 1$)$.

\begin{tabular}{|l|c|c|}
\hline \multirow{3}{*}{ Gender } & & Percentage \\
\hline \multirow{5}{*}{ Age (yrs.) } & Male & $45 \%$ \\
\cline { 2 - 3 } & Female & $55 \%$ \\
\cline { 2 - 3 } & $0-5$ & $34 \%$ \\
\cline { 2 - 3 } & $6-10$ & $9 \%$ \\
\cline { 2 - 3 } & $11-15$ & $4 \%$ \\
\cline { 2 - 3 } & $16-24$ & $9 \%$ \\
\cline { 2 - 3 } & $25-34$ & $18 \%$ \\
\cline { 2 - 3 } & $35-44$ & $14 \%$ \\
\cline { 2 - 3 } & $45-54$ & $6 \%$ \\
\cline { 2 - 3 } & $55-64$ & $3 \%$ \\
\hline
\end{tabular}

Table 1: Demographic characteristics of the sample:

\section{Proportions of URTIs}

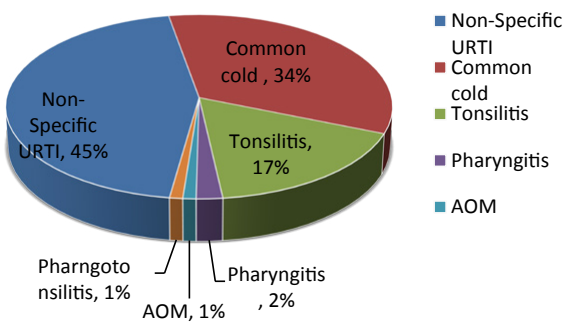

Figure 1: Distribution of the type of URTIs diagnoses $(n=385)$.

\begin{tabular}{|l|c|}
\hline Antibiotics & Percentage \\
\hline 1. Amoxicillin & $65.5 \%$ \\
\hline 2. Phenoxymethyl penicillin & $22.0 \%$ \\
\hline 3. Azithromychin & $4.2 \%$ \\
\hline 4. Benzathine penicillin & $2.6 \%$ \\
\hline 5. Cefuroxime & $1.9 \%$ \\
\hline 6. Ceftriaxone & $1.6 \%$ \\
\hline 7. Erythromycin & $1.6 \%$ \\
\hline 8. Cloxacillin & $0.3 \%$ \\
\hline
\end{tabular}

Table 2: Most prescribed antibiotics.

\begin{tabular}{|l|c|}
\hline Rates & Percentage \\
\hline Antibiotic prescribing & $95 \%(95 \% \mathrm{Cl} 90 \%, 98 \%)$ \\
\hline Non-specific URTIs & $100 \%(95 \% \mathrm{Cl} 100 \%, 100 \%)$ \\
\hline Tonsillitis & $42 \%(95 \% \mathrm{Cl} \mathrm{34 \% ,51 \% )}$ \\
\hline Common cold & $89 \%(95 \% \mathrm{Cl} 52 \%, 100 \%)$ \\
\hline Pharyngitis & $100 \%(95 \% \mathrm{Cl} 100 \%, 100 \%)$ \\
\hline Pharyngotonsillitis & $100 \%(95 \% \mathrm{Cl} 100 \%, 100 \%)$ \\
\hline
\end{tabular}

Table 3: Type of URTIs influenced antibiotic prescribing.

The most common diagnosis was non-specific URTI 45\% ( $\mathrm{n}=174)$ followed by the 'common cold' $34 \%(\mathrm{n}=130)$, then tonsillitis $17 \%$ $(n=67)$, pharyngitis $2 \%(n=9), A O M 1 \%(n=4)$ and pharyngitis and tonsillitis $1 \%(n=4)$.

Seventy-eight per cent of prescriptions included an antibiotic (302/385: 95\% CI, 74\%-82\%). Further bivariate analysis of antibiotic prescribing (response variable) and illness, age, gender and profession (exposure variables) revealed that age and illness were significantly associated with antibiotic prescribing $(\mathrm{p}<0.05)$ while gender and profession rank were not (Table 2).

The study also revealed that the type of URTI influenced antibiotic prescribing $\left(\mathrm{X}^{2}=143.790, \mathrm{p}<0.05\right)$ (Table 3$)$. It was also observed that the rate of antibiotic prescribing in children was higher than that of adults, that is, $90 \%(95 \%$ CI $84,94 \%)$ and $68 \%(95 \%$ CI $62 \%, 75 \%)$ respectively, and children were more likely to receive an antibiotic prescription than adults $\left(\mathrm{x}^{2}=27.073, \mathrm{p}<0.05\right)$. The rate of antibiotic prescribing in male patients was $78 \%(95 \%$ CI $71,84 \%)$ and that for female patients was $79 \%$ (95\% CI 73, 84\%). However, there was no significant relationship between gender and likelihood of antibiotic prescribing $\left(\mathrm{X}^{2}=0.53, \mathrm{p}>0.05\right)$. The study also showed that General Practitioners (GPs) more frequently prescribed antibiotics for patients with URTIs than nurses, $61 \%(n=185)$ and $39 \%(n=117)$ respectively, but prescriber profession did not significantly influence antibiotic prescribing $\left(\mathrm{X}^{2}=0.695, \mathrm{p}>0.05\right)$. 
Page 4 of 7

\section{Rationality (compliance to STGS) of Antibiotic Prescribing}

The overall compliance to standard treatment guidelines (STGs) was only $47 \%$ (95\% CI 40\%, 54\%). As shown in Figure 2, antibiotic treatment compliance for the individual illnesses was as follows: $11 \%$ $(\mathrm{n}=1)$ of prescriptions for patients with pharyngitis, $36 \%(\mathrm{n}=24)$ for tonsillitis, $25 \%(\mathrm{n}=1)$ for pharyngotonsillitis, $0 \%$ for AOM and only $58 \%$ of prescriptions for patients with the common cold while $42 \%(n=55)$ of these common cold prescriptions had an antibiotic (Figure 3 ).

\section{Cost of Rational and Irrational Antibiotic Prescribing}

The total cost of antibiotic prescribing in this study was $\mathrm{N} \$ 2$, 406.98 of which antibiotics in non-specific URTIs accounted for $48 \%$ total cost $(\mathrm{N} \$ 1,150.45)$. The total cost of antibiotics prescribed for the common cold, tonsillitis, pharyngitis, pharyngotonsillitis and AOM was $\mathrm{N} \$ 1,256.53$. The rationally prescribed antibiotics cost N\$204.46 while the irrationally prescribed ones cost N\$1052.07. According to this, irrational antibiotic prescribing cost is five times more than the rationally prescribed (Tables 4 and 5).

\section{Discussion}

The purpose of this study was to explore antibiotic prescribing in patients with URTIs in order to gain information about the use of these medicines in order to develop strategies and make recommendations that will promote judicious use of these important medicines. URTIs are viral in $80 \%$ of the cases and do not respond to antibiotic therapy [3] but physicians in many settings frequently prescribe antibiotics for these illnesses [4]. Therefore, URTIs are important targets for strategies aimed at reducing excess antibiotic use [12].

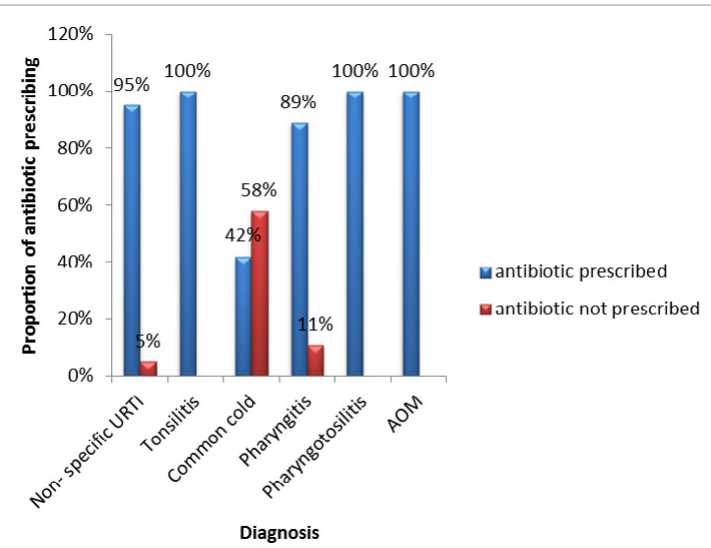

Figure 2: Distribution of antibiotic prescribing according to types of URTIs.

TREATMENT COMPLIANCE TO STGs

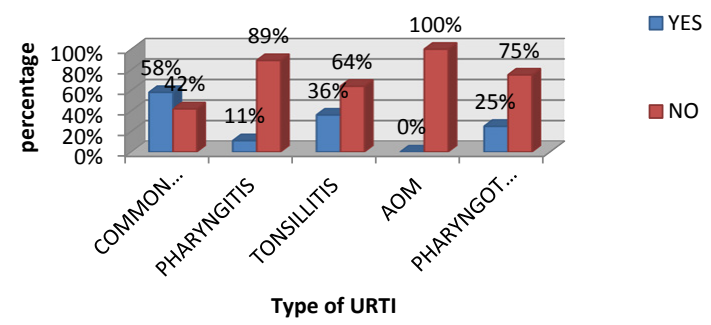

Figure 3: Proportions of rational treatment of URTIs according to STGs by illness or condition.

\begin{tabular}{|l|c|c|c|c|c|}
\hline \multirow{2}{*}{ Name of Antibiotic } & $\begin{array}{c}\text { Dosage } \\
\text { form }\end{array}$ & $\begin{array}{c}\text { Quantity: } \\
\text { Rational }\end{array}$ & $\begin{array}{c}\text { Rational } \\
\text { Cost } \\
\text { (N\$) }\end{array}$ & $\begin{array}{c}\text { Quantity: } \\
\text { irrational }\end{array}$ & $\begin{array}{c}\text { Irrational } \\
\text { Cost } \\
\text { (N\$) }\end{array}$ \\
\hline \multirow{2}{*}{ Amoxicillin } & Suspension & 0 & 0 & 32 & 172.48 \\
\hline \multirow{2}{*}{ Phenoxymethylpenicillin } & Capsules & 0 & 0 & 980 & 156.47 \\
\cline { 2 - 6 } & Suspension & 12 & 86.04 & 32 & 229.44 \\
\hline Erythromycin & Tablets & 456 & 90.76 & 284 & 56.53 \\
\hline Cloxacillin & Tablets & 0 & 0 & 120 & 49.51 \\
\hline Azithromycin & Capsules & 0 & 0 & 40 & 9.19 \\
\hline Cefuroxime & Tablets & 3 & 27.66 & 9 & 82.98 \\
\hline Cotrimoxazole & Suspension & 0 & 0 & 2 & 108.56 \\
\hline & Tablets & 0 & 0 & 48 & 67.12 \\
\hline Benzathine penicillin inj & Suspension & 0 & 0 & 1 & 56.42 \\
\hline ceftriaxone inj & Tablets & 0 & 0 & 20 & 2.74 \\
\hline Total & Injection & 0 & 0 & 8 & 52.02 \\
\hline & Injection & 0 & 0 & 3 & 8.61 \\
\hline & - & - & 204.46 & - & 1052.07 \\
\hline
\end{tabular}

Table 4: Comparison of the cost of rational and irrational antibiotic prescribing.

\begin{tabular}{|c|c|c|c|}
\hline Name of Antibiotic & Dosage form & Quantity dispensed & Cost $\mathbf{( \$ N})$ \\
\hline \multirow{2}{*}{ Amoxicillin } & Capsules & 2520 & 402.34 \\
\cline { 2 - 4 } & Suspension & 57 & 307.23 \\
\hline \multirow{2}{*}{ Phenoxymethylpenicillin } & Tablets & 128 & 22.50 \\
\cline { 2 - 4 } & Suspension & 4 & 28.68 \\
\hline \multirow{2}{*}{ Erythromycin } & Tablets & 80 & 32.80 \\
\hline \multirow{2}{*}{ Azithromycin } & Tablets & 18 & 165.96 \\
\cline { 2 - 4 } & Suspension & 1 & 58.28 \\
\hline \multirow{2}{*}{ Cefuroxime } & Tablets & 48 & 67.12 \\
\hline \multirow{2}{*}{ Cotrimoxazole } & Suspension & 1 & 56.42 \\
\hline Total cost & Suspension & 1 & 9.12 \\
\hline
\end{tabular}

Table 5: Cost of antibiotic prescribing in non-specific URTIs.

Seventy eight (78\%) $(95 \%$ CI $74 \%, 82 \%)$ was the rate of antibiotic prescribing in this study. This rate was notably high and a cause of serious concern. The rate of antibiotic prescribing for patients with URTIs at $\mathrm{KHC}$ was $78 \%$ (95\% CI, $74 \%-82 \%)$. The rate was higher in children $90 \%$; CI, 84\%-94\%) than in adults (68\%; CI, 62\%-75\%) respectively). In male patients the rate was $78 \%$ (CI, 71-84\%) and in female patients it was 79\% (CI, 73-84\%). Further analysis with the chisquare test of, antibiotic prescribing (response variable) and illness, age, gender and profession (exposure variables) showed that age and illness were significantly associated with antibiotic prescribing $(\mathrm{p}<0.05)$ while gender and profession rank were not.

Compliance to treatment guidelines was only $47 \%$ (CI, 40\%$54 \%)$. The overall cost of antibiotic prescribing in this study was to N\$ 2406.98 of which non-specific URTIs accounted for 48\% (N\$1150.45; irrationally prescribed antibiotics, $44 \%(\mathrm{~N} \$ 1052.07)$ and those that were rationally prescribed accounted for only $8 \%$ (N\$204.46). The total cost of antibiotic treatment of illnesses assessed for rationally was $\mathrm{N} \$ 1256.5$. N\$ 1052.07 (84\%) was the cost of irrationally prescribed antibiotics N\$204.46 (16\%), showing that the cost of irrational prescribing was cost five times more than that of rational prescribing.

Antibiotic over-prescribing is a problem in many countries and this result shows that Namibia is not an exception. In a study conducted in Eritrea, it was confirmed that $75 \%$ of the adults and children diagnosed with URTI were prescribed antibiotics [21] and in Malaysia, the rate of antibiotic prescribing for URTIs was $64.8 \%$ [22]. 
About forty-two per cent (42\%) (95\% CI 34\%, 51\%) of patients with the common cold were prescribed an antibiotic. This rate is a cause for concern because prescribing antibiotics for common cold in adults and children does not have any therapeutic benefits, but only increases the risk of developing side effects, especially in adults. In addition, antibiotics do not warrant a better outcome in terms of cure or persistence of symptoms in patients that receive antibiotics compared to those that do not [23].

The rate for non - specific URTIs was 95\% (95\%CI 90\%, 98\%). URTIs are typically acute viral infections. Guidelines by Centre of Disease Control (CDC) do not recommend antibiotic prescribing in non-specific URTIs because antibiotics neither enhance illness resolution nor prevent complications [24]. This high rate in Namibia could be attributed to a lack of specifications in the treatment guidelines on how to manage nonspecific URTIs and whether antibiotics should be prescribed or not.

All four (4) (100\%) patients with AOM were treated with an antibiotic in conformity with Namibia Standard Treatment Guidelines (NSTG). However, viruses (especially respiratory syncytial virus and influenza) can also cause AOM without co-infection with bacteria [25]. Since the NSTGs only give antibiotic therapy as the only treatment option could be promoting irrational antibiotic prescribing in some of the cases that are viral. According to the CDC guidelines [24] Acute Otitis Media does not always require antibiotic therapy, but observation is an acceptable option in healthy children with mild symptoms. This option is appropriate only when follow-up can be ensured and antibacterial agents started if symptoms persist or worsen.

About eight (89\%) patients with pharyngitis, 67 (100\%) with tonsillitis and $4(100 \%)$ with pharyngotonsillitis had an antibiotic/s prescribed. The Namibia Standard Treatment Guidelines [26] recommend antibiotic therapy and analgesics for patients with tonsillitis/ pharyngitis or both which could explain the high rate of antibiotic prescribing in these conditions. These illnesses could also be viral. For example, acute pharyngitis is caused Group A B Hemolytic Streptococcus (GABHS) in only $5-15 \%$ of adults implying that the large majority of adults have a self-limiting illness that can be managed supportively. In addition, only $15 \%$ of pharyngitis is caused by GABHS and most of them are viral in children. In order to promote rational antibiotic prescribing in adult patients, the CDC recommends that antibiotic prescribing be limited to those patients with the highest likelihood of GABHS using clinical decision criteria. This involves screening of all adults with pharyngitis for the presence of 4 main criteria which are history of fever, tonsillar exudates, no cough and tender anterior cervical lymphadenopathy. Testing is only recommended for patients with two or more of the same criteria. These patients should be tested using a rapid antigen test and antibiotic therapy limited to patients with a positive test. Treating of patients with none or only one of these criteria is not recommended because these patients are unlikely to have a bacterial infection [24].

Antibiotic over-prescribing entails an increase in cost, side effects, and the emergence and spread of resistant bacteria. These bacteria become resistant to affordable and effective first line antibiotics, a situation that is worsened by the fact that the development of new generations of antibiotics is not promising [27]. Some of the reasons that lead to antibiotic over use are diagnostic uncertainty, time pressure, patient demand and expectations [28]. Gonzales et al. [29] suggested that unnecessary adverse effects of antibiotics and the evolution of antimicrobial resistance can be reduced by judicious use of these drugs. Some of the proven ways that help reduce antibiotic over-prescribing in PHC are patient education and delayed antibiotic prescribing. Patient education through providing leaflets that describes the nature and expected course of their illness and discloses the uncertainty of benefit from treatment with antibiotics [30]. Delaying antibiotic use by giving the patient a prescription and instructing them only to have it filled if symptoms do not resolve is also an effective means of reducing antibiotic use for acute respiratory infections [31].

Compliance to STGs was $47 \%$ (95\% CI $40 \%, 54 \%$ ). This result is similar to those of other studies, for example, a study in the United States showed a $57 \%$ overall adherence to guidelines for pharyngitis, URI and acute sinusitis [32]. Failure to prescribe according to clinical guidelines is one of the problems associated with the irrational use of medicines. The low compliance to guideline for individual illnesses in this study (common cold $=58 \%$, pharyngitis $=11 \%$, tonsillitis $=36 \%$, $\mathrm{AOM}=0 \%$ and pharyngotonsillitis $=25 \%$ ), indicates a high prevalence of irrational prescribing. Furthermore, if antibiotic treatment is indicated for $\mathrm{AOM}$ and pharyngitis or tonsillitis, compliance to guidelines is very important to avoid the risk of complications, treatment failure and the development of antibiotic resistance. This can occur if the doses, duration of treatment and frequency are different from the recommended.

$10-40 \%$ of national health budgets are spent on medicines and if medicines are not prescribed [33]. For example, $\$ 1.1$ billion is spent annually on unnecessary adult upper respiratory infection antibiotic prescriptions [24]. In this study, the rationally prescribed antibiotics cost $\mathrm{N} \$ 204.46$ while the irrationally prescribed ones cost $\mathrm{N} \$ 1052.07$. According to this, irrational antibiotic prescribing cost five times more than the rationally prescribed. This result shows that irrational antibiotic prescribing leads to wastage of resources. The overall rate of antibiotic prescribing in this study (78\%) is a cause for serious concern because these infections are viral in $80 \%$ of the cases. The low compliance (treatment inappropriateness) to STGs of antibiotic prescriptions for the treatment of URTIs (47\%) could explain the high cost (N\$1052.07) of irrationally prescribed antibiotics that cost five times more than the rationally prescribed antibiotics $(\mathrm{N} \$ 204.46)$.

Future research should focus on determining factors associated with antibiotic over prescribing in patients with URTIs as well as factors associated with non-compliance to standard treatment guidelines.

\section{Recommendations}

The study revealed very important information on which basis recommendations can be made. These recommendations included the following:-

In order to reduce the irrational use of antibiotic prescribing in URTIs, it is of utmost importance that at the health facility level, the health care providers are trained to know as when to prescribe antibiotic. This will assist in alleviating antibiotic resistance. Health workers continuing education should be strengthened through workshops. Equally important is the periodic antibiotic use audit, which can provide feedback to prescribers in health facilities on antibiotic use expenditure and resistance patterns.

MoHSS should consider adopting a strategy of delayed antibiotic use, which has shown effectiveness in reducing antibiotic usage for URTIs. This strategy Involves giving the patient a prescription and clearly instructing them only to have it filled if symptoms do not resolve [31].

The dosing for phenoxymethyl penicillin in children should be revised to adjust by weight. The duration of treatment of tonsillitis 
should be changed to 10 days to achieve maximal GABHS eradication rates as recommended by WHO.

The MOHSS should consider adopting the CDC clinical decision criteria for prescribing antibiotics. This can be done by screening all patients with pharyngitis for the presence of 4 centre criteria: history of fever, tonsillar excudates no cough and tender anterior cervical lymphadenopathy. It helps when there is presence of two or more of these symptoms as an indication of a high likelihood a bacterial infection. In order to confirm the diagnosis, a rapid streptococcal antigen test is recommended to prescribe the appropriate antibiotic. It is recommended that the MOHSS make streptococcal test kits available in all health facilities.

MoHSS should consider purchasing cough syrups (antitussives and expectorants). Though they have doubtful efficacy, these could help reduce antibiotics prescribing by giving a placebo effect to patients.

Cough syrups can be the first line choice in patients with URTIs especially in cases where bacterial infection is unlikely or has been excluded, for example the common cold and non-specific URTIs. In addition, these cough syrups also contain antihistamines, analgesics, etc. that help alleviate symptoms of URTIs. Having these in state facilities will also prevent prescribers from rushing into prescribing antibiotics for even conditions like common cold that do not require antibiotics. Even though the cost of these cough syrups could raise concerns, the cost of antibiotic resistance is even higher.

The study also recommends awareness to rise about antibiotics among patients and the community. Strategies that can be used cover a wide range of activities, such as information leaflets, radio talks, media campaigns as well as television and rational use of antibiotics. Furthermore, further research is recommended to identify factors contributing to antibiotic over-prescribing in URTIs in Namibia. This research will also identify barriers of compliance to standard treatment guidelines.

\section{Study Limitations}

The study was conducted at one health facility. This prevented generalisation of the results to the larger population of Windhoek or Namibia.

In this study, the NSTGs of 2011 were intended to be used to assess compliance to the recommended treatment for pharyngitis or tonsillitis in children. This is because the NSTG do not specify dosing in children by weight. Hence, the WHO recommendations of 2001 were also used to access rationality of prescriptions with doses by weight for children.

This study did not confirm the accuracy of diagnosis in terms of correlating symptoms, diagnosis and treatment. To remedy this shortcoming, further research is needed to identify which subsets of respiratory tract infection and correlating symptoms and signs are more likely to result in the prescription of antibiotic.

Meaningful statistical conclusion on compliance to STGs for AOM, pharyngitis and pharyngeotosilitis could not be made because of the small number of prescriptions with such diagnoses.

\section{References}

1. Bauman KA (2000) The Family Physician's Reasonable Approach to Upper Respiratory Tract Infection Care for this century. Archives of Family Medicine 9: 596-597.

2. Kontarakis N, Tsiligianni IG, Papadokostakis P, Giannopoulou E, Tsironis L, et al. (2011) Antibiotic prescriptions in primary health care in a rural population in Crete, Greece. BMC Res Notes 4: 38
3. Mazur E (2010) Rational antibiotic therapy of acute upper respiratory tract infections. PolMerkurLekarski 29: 304-308.

4. Shlomo V, Adi R, Eliezer K (2003) The knowledge and expectations of parents about the role of antibiotic treatment in upper respiratory tract infection; a survey among parents attending the primary physician with their sick child. BMC family practice $4: 20$.

5. Leblebicioglu H, Canbaz S, Peksen Y, Gunaydin M (2002) Physicians' Antibiotic Prescribing Habits for Upper Respiratory Tract Infections in Turkey. Journal of chemotherapy 14: 181-184.

6. http://www.who.int/mediacentre/factsheets/fs338/en/

7. Gould IM (2009) Antibiotic resistance: the perfect storm. International Journal of antimicrobial agents $34: 52-55$.

8. Hashemi S, Nasrollah A, Rajabi M (2013) Irrational Antibiotic Prescribing: a local Issue or Global concern. EXCLI journal 12: 384-395.

9. Togoobaatar G, Ikeda N, Ali M, Sonomjamts M, Dashdemberel S, et al. (2010) Survey of non-prescribed use of antibiotics for children in an urban community in Mongolia. Bulletin of the World health Organization 88: 930-936.

10. Erika MC, Myrielle D, Pierre M, Damien O, Shigui R (2008) The Impact of Different Antibiotic Regimens on the Emergence of Antimicrobial-Resistant Bacteria. PLoS ONE 3: e4036.

11. Phulu B, Sumbi V, Lates, JA (2011) National Pharmacy Management Information Systems (PMIS) Feedback report. Windhoek. Ministry of Health and Social Services.

12. Harris RH, Mac Kenzie TD, Leeman-Castillo B, Corbett KK, Batal AH, et al. (2003) Optimizing Antibiotic Prescribing for Acute Respiratory Tract Infections in an Urban Urgent Care Clinic. Journal of General Internal Medicine 18: 326 334

13. http://www.who.int/medicines/areas/rational_use/en/

14. Walley J, Wright $\mathrm{J}$ (2010) Public Health: An action guide to improving health (2 ${ }^{\text {nd }}$ edn), Oxford University Press Inc, New York 216.

15. Jones R, Britten N, Culpepper L, Gass AD, Grol R, et al. (2005) Oxford Textbook of Primary Medical Care. New York: Oxford University Press.

16. Rohilla A, Sharma V, Kumar S (2013) Upper Respiratory Tract Infections: An overview. International Journal of Current Pharmaceutical Research 5: 1-3.

17. Cotton MF, Innes S, Jaspan H, Madide A, Rabie H (2008) Management of upper respiratory tract infections in children. South Africa Family practice, 50: 6-12

18. Brink HI, Van der Walt C, Van Rensburg G (2006) Fundamentals of research methodology for health Care Professionals. Juta and Company Ltd.

19. Antonisamy B, Christopher S, Samuel PP (2010) Biostatistics: Principles and Practice. Tata McGraw-Hill Education Private Limited. New Delhi.

20. Suttajit S, Wagner AK, Tantipidoke R, Ross-Degnan D, Sitthi-Amorn C (2005) Patterns, appropriateness, and predictors of antimicrobial prescribing for adults with upper respiratory infections in urban slum communities of Bangkok. The south east journal of tropical medicine and public health 36: 489-97.

21. http://apps.who.int/medicinedocs/pdf/s6160e/s6160e.pdf.

22. Kho BP, Ong CM, Tan FT, Wee CY (2013) Antibiotic prescribing for upper respiratory tract infections in Sarawak district hospitals. Medical journal of Malaysia 68: 136-40.

23. Arroll B, Kenealy T (2013) Antibiotics for common cold and acute purulent rhinitis. Cochrane Database of Systematic reviews 4(6): CD000247.

24. http://www.cdc.gov/getsmart/campaign-materials/treatment-guidelines.html.

25. Morris PS, Leach PA (2009) Acute and chronic otitis media. Pediatrict clinics of North America 56: 1383-1399.

26. Namibian Standard Treatment Guidelines (2011) Ministry of Health and social services. Windhoek, 259,276-277.

27. Ivanovoska V, Holloway KA (2013) Interventions to Improve Antibiotic Prescribing in Upper Middle Income Countries: A Systematic Review of the Literature 1990 -2009. Macedonian Journal of Medical Sciences 6: 84-91.

28. http://blogs.cdc.gov/safehealthcare/2010/11/11/antibiotic-prescriptions$\%$ E2\%80\%93-where-you-live-may-matter/

29. Gonzales R, Bartlett JG, Besser RE, Hickner JM, Hoffman JR, et al. (2001) 
Citation: Kunda M, Haoses-Gorases L, Goraseb M (2015) An Investigation of Antibiotic Prescribing in Patients with Upper Respiratory Tract Infections (Urtis) at Katutura Health Centre, Windhoek, Namibia. Single Cell Biol 4: 122. doi:10.4172/2168-9431.1000122

Principles of appropriate use of antibiotics for treatment of nonspecific upper respiratory tract infections in adults: background. Annals of internal medicine 134: 490-494.

30. Farquhar D (2002) Reducing antibiotic use for acute bronchitis by giving patients written information. Canadian Medical Association Journal 166: 776.

31. Arroll B, Keanely T, Kerse N (2003) Do delayed prescriptions reduce antibiotic use in respiratory tract infections? A systematic review. The British Journal of general Practice 53: 871-877

32. Crocker A, Alweis R, Scheirer J, Schamel S, Wasser T, et al. (2013) Factors affecting adherence to evidence-based guidelines in the treatment of URI, sinusitis, and pharyngitis. Journal of Community Hospital Internal Medicine Perspectives 3: 20744.

33. http://www.who.int/mediacentre/factsheets/fs338/en/ 